\title{
Antigen-specific expansion of human regulatory $T$ cells as a major tolerance mechanism against mucosal fungi
}

\author{
P Bacher ${ }^{1}$, O Kniemeyer ${ }^{2,3}$, A Schönbrunn ${ }^{4}$, B Sawitzki $^{4}$, M Assenmacher $^{5}$, E Rietschel $^{6}$, A Steinbach ${ }^{7}$, \\ OA Cornely ${ }^{7,8}$, AA Brakhage ${ }^{2}$, A Thiel $^{4}$ and A Scheffold ${ }^{1,9}$
}

Foxp3 ${ }^{+}$regulatory Tcells (Treg) have a central role for keeping the balance between pro- and anti-inflammatory immune responses against chronically encountered antigens at mucosal sites. However, their antigen specificity especially in humans is largely unknown. Here we used a sensitive enrichment technology for antigen-reactive T cells to directly compare the conventional vs. regulatory $\mathrm{CD} 4^{+} \mathrm{T}$-cell response directed against two ubiquitous mucosal fungi, Aspergillus fumigatus and Candida albicans. In healthy humans, fungus-specific CD $4{ }^{+} \mathrm{CD} 25^{+} \mathrm{CD} 127^{-}$Foxp ${ }^{+}$Treg are strongly expanded in peripheral blood and possess phenotypic, epigenetic and functional features of thymus-derived Treg. Intriguingly, for A. fumigatus, the strong Treg response contrasts with minimal conventional T-cell memory, indicating selective Treg expansion as an effective mechanism to prevent inappropriate immune activation in healthy individuals. By contrast, in subjects with $A$. fumigatus allergies, specific Th2 cells were strongly expanded despite the presence of specific Treg. Taken together, we demonstrate a largely expanded Treg population specific for mucosal fungi as part of the physiological human T-cell repertoire and identify a unique capacity of $A$. fumigatus to selectively generate Treg responses as a potentially important mechanism for the prevention of allergic reactions.

\section{INTRODUCTION}

The mucosal immune system is constantly confronted with the challenge to combat a vast number of pathogens from the environment without causing collateral tissue damage due to uncontrolled or inappropriate immune reactions. There is substantial evidence from animal experiments that regulatory $\mathrm{T}$ cells (Treg) have an important role in maintaining tolerance against commensals or inhaled antigens encountered at mucosal sites. ${ }^{1-6}$ However, the antigen specificity of this protective Treg population in particular in humans remained so far elusive due to the high number of potential targets and a lack of specific and sensitive technologies for the direct quantitative and qualitative analysis of Treg with a defined antigen specificity. ${ }^{7}$
Fungi represent a prototypic example for mucosally encountered environmental pathogens and/or commensal microbiota whose importance has been underestimated for many years. ${ }^{8}$ Humans continuously inhale several thousands of airborne fungal spores per day. ${ }^{9,10}$ Other fungi such as Candida albicans belong to the normal mucosal microflora of humans. ${ }^{11}$ In immunocompromised patients, fungi such as Aspergillus fumigatus and Candida albicans can cause life-threatening invasive infections. ${ }^{12}$ In addition, inhaled airborne fungi can cause allergic hypersensitivity especially in patients with respiratory disorders, such as severe asthma or cystic fibrosis (CF). These patients frequently suffer from severe Th2-driven allergic reactions and allergic bronchopulmonary aspergillosis (ABPA). ${ }^{13}$

\footnotetext{
${ }^{1}$ Department of Cellular Immunology, Clinic for Rheumatology and Clinical Immunology, Charité - University Medicine, Berlin, Germany. ${ }^{2}$ Department of Molecular and Applied Microbiology, Leibniz Institute for Natural Product Research and Infection Biology - Hans-Knoell Institute (HKI) Jena and Friedrich Schiller University Jena, Jena, Germany. ${ }^{3}$ Integrated Research and Treatment Center, Center for Sepsis Control and Care Jena, University Hospital (CSCC), Jena, Germany. ${ }^{4}$ Regenerative Immunology and Aging, Berlin-Brandenburg Center for Regenerative Therapies, Charité - University Medicine, Berlin, Germany. ${ }^{5}$ Miltenyi Biotec GmbH, Bergisch Gladbach, Germany. ${ }^{6} \mathrm{CF}$ Centre Cologne, Children's Hospital, University of Cologne, Cologne, Germany. ${ }^{7}$ Department I of Internal Medicine, University Hospital of Cologne, Cologne, Germany. ${ }^{8}$ Clinical Trials Center Cologne ZKS Köln (BMBF 01KN1106), Cologne Excellence Cluster on Cellular Stress Responses in Aging-Associated Diseases (CECAD), and Center for Integrated Oncology ClO Köln-Bonn, University of Cologne, Cologne, Germany and ${ }^{9}$ German Rheumatism Research Centre (DRFZ) Berlin, Leibniz Association, Berlin, Germany. Correspondence: A Scheffold (alexander.scheffold@charite.de)
} 
Strikingly, despite daily contact with fungal antigens, immunocompetent persons are highly resistant to severe fungal infections as well as hypersensitivity reactions. ${ }^{14}$ This implicates the existence of efficient protective immune responses as well as regulatory mechanisms that are able to prevent inappropriate immune reactions. ${ }^{15}$ It has been shown that fungal components trigger efficient innate immune reactions and eventually also initiate adaptive immune responses mainly mediated by $\mathrm{CD} 4{ }^{+} \mathrm{T}$ cells. ${ }^{16,17}$ We recently showed that fungus-reactive conventional memory $\mathrm{CD} 4^{+}$ $\mathrm{T}$ cells are indeed present in peripheral blood of all healthy donors $^{18}$ (and unpublished data), which most likely reflects the ubiquitous presence of fungal antigens in the environment. However, the cellular basis for anti-inflammatory or tolerogenic mechanisms, which avoid immunopathology such as chronic inflammation or allergies against fungi is less clear. We therefore analyzed Treg as well as conventional naive and memory CD4 ${ }^{+} \mathrm{T}$ cells (Tcon) specific for the two major fungal pathogens A. fumigatus and C. albicans directly in blood of human subjects. This comparison was possible by using a new sensitive pre-enrichment technology for the direct detection of rare antigen-specific Tcon and Treg, according to the converse expression pattern of the activation markers CD154 and CD137. ${ }^{18,19}$ Interestingly, we found that both fungi, but in particular A. fumigatus, elicited a strong Treg response in adult but not in cord blood. For C. albicans, the specific Treg response was accompanied by a parallel dominant memory Tcon (Tmem) formation. However, for A. fumigatus, the balance was strongly shifted toward Treg as the specific Tcon were composed of only a minimally expanded memory and a large naive compartment. Thus, Treg numbers even exceeded those of Tmem, resulting in a marked suppression of the anti- $A$. fumigatus Tcon response in in vitro assays. This presumably protective Treg dominance was abrogated in CF patients with Aspergillus-associated allergies due to a strong expansion of Th2-type Tmem. These data provide direct evidence for the preferential generation of an antigen-specific Treg response against a chronically encountered mucosal pathogen and suggest that subversion or bypassing of this mechanism contribute to the development of allergies.

\section{RESULTS}

\section{High frequencies of fungus-specific memory Treg in peripheral blood of healthy donors}

To better define the contribution of $\mathrm{T}$ cells to the delicate balance between pro- and anti-inflammatory immune responses against chronically exposed fungi, we performed a side-by-side comparison of human Tcon and Treg responses against A. fumigatus and C. albicans. Converse expression of CD137 and CD154 following short-term stimulation with fungal lysates enabled identification of activated forkhead box protein P3 (Foxp3) expressing Treg together with Tcon. ${ }^{19}$

Stimulation with $A$. fumigatus and C. albicans antigens induced overall low frequencies of $\mathrm{CD} 154^{+}$or $\mathrm{CD} 137^{+} \mathrm{CD} 4^{+}$ $\mathrm{T}$ cells (Figure 1a and $\mathbf{b}$ ). Despite their low overall abundance, this corresponds to surprisingly high frequencies within the
$\mathrm{CD} 25^{+}$Foxp $3^{+}$population, which actually represents only $5-10 \%$ of the total $\mathrm{CD} 4^{+} \mathrm{T}$ cells, i.e., $1.3 \%$ (range $0.06-3.4 \%$ ) for A. fumigatus and $0.6 \%$ for C. albicans (range $0.02-2.6 \%$ ) (Figure 1c). In contrast, the frequencies of fungus-reactive Tcon among total $\mathrm{CD} 4{ }^{+} \mathrm{T}$ cells were about 5 - to 10 -fold lower, and here the frequencies of $C$. albicans-reactive Tcon were significantly higher $(0.27 \%$; range $0.11-0.55 \%)$ than those of A. fumigatus-reactive Tcon (0.19\%; range $0.06-0.37 \%)$.

For a more sensitive and detailed analysis, we used antigenreactive T-cell enrichment (ARTE) ${ }^{7,18}$ to enable the quantification and characterization of the few $\mathrm{CD} 154^{+}$and $\mathrm{CD} 137^{+}$ cells from larger cell samples $\left(\geq 1 \times 10^{7} \mathrm{PBMC}\right)$ with high sensitivity and specificity (Figure 1d). Approximately 90\% (range $60-93 \%$ ) of the CD137 ${ }^{+}$cells were positive for Foxp3 and CD25 and negative for CD127 (Figure 1d and e). In addition, the majority of the antigen-reactive $\mathrm{CD} 137^{+}$ CD $154^{-}$Foxp $^{+}$cells co-expressed the transcription factor Helios (Figure 1f). FACS-sorted CD137 ${ }^{+} \mathrm{CD} 154^{-}$cells had a highly demethylated Treg-specific demethylated region (TSDR), at levels comparable to $\mathrm{CD} 25^{+} \mathrm{CD} 127^{\mathrm{dim}}$-sorted $\mathrm{CD}^{+} \mathrm{T}$ cells or Staphylococcal enterotoxin B (SEB)stimulated $\mathrm{CD} 137^{+} \mathrm{CD} 154^{-} \mathrm{CD} 4^{+}$cells. In contrast, $\mathrm{CD} 154^{+}$ CD137 ${ }^{-}$T cells had a methylated Foxp3 locus (Figure 1g) and a phenotype matching that of Tcon (Foxp3 ${ }^{-} \mathrm{CD} 25^{-}$ $\mathrm{CD} 127^{+}$).

Analysis of CD45RO and CCR7 expressions on the fungusspecific $\mathrm{CD} 137^{+} \mathrm{CD} 154^{-}$Treg revealed a memory phenotype with a higher frequency of cells in the CCR7 ${ }^{-}$effector memory compartment (Figure 2a and b). Further analysis of cytokine expression demonstrated that production of the cytokines IL-2, IL-10, IL-17, TNF- $\alpha$ and IFN- $\gamma$ is restricted to the fungusspecific CD154 ${ }^{+}$Tcon, but lacking in $\mathrm{CD} 137^{+}$Treg (Figure 2c and Supplementary Figure S1 online).

In summary, these data confirm that the combined analysis of $\mathrm{CD}_{137^{+}}$and $\mathrm{CD} 154^{+}$expression can identify fungusspecific memory Treg as well as Tcon in human peripheral blood. The direct comparison between both fungi further suggests that A. fumigatus has a stronger potential to induce and/or expand Treg, whereas C. albicans induces a stronger Tcon response.

\section{Fungus-reactive $\mathrm{CD} 137^{+}$Treg can be expanded and maintain strong antigen-specific suppressive potential}

To proof specificity and function of the fungus-specific Treg, we expanded magnetically enriched $\mathrm{CD} 137^{+} \mathrm{T}$ cells in the presence of IL-2 and rapamycin for up to 3 weeks. To determine specific reactivity, CD137 expression was analyzed upon rechallenge with the specific or control antigens in the presence of autologous APCs. As shown in Figure 3a, fungus-specific Treg specifically upregulated $\mathrm{CD}_{13} 7^{+}$expression after restimulation with the specific fungal lysate but not in response to irrelevant antigens. In contrast, polyclonally expanded CD25 ${ }^{+}$ CD127 $7^{\mathrm{dim}}$ Treg reacted strongly only upon stimulation with the high control SEB. Interestingly, we also saw low reactivity against A. fumigatus in the polyclonal expanded Treg cell lines (Figure 3a), which is consistent with the high frequency 

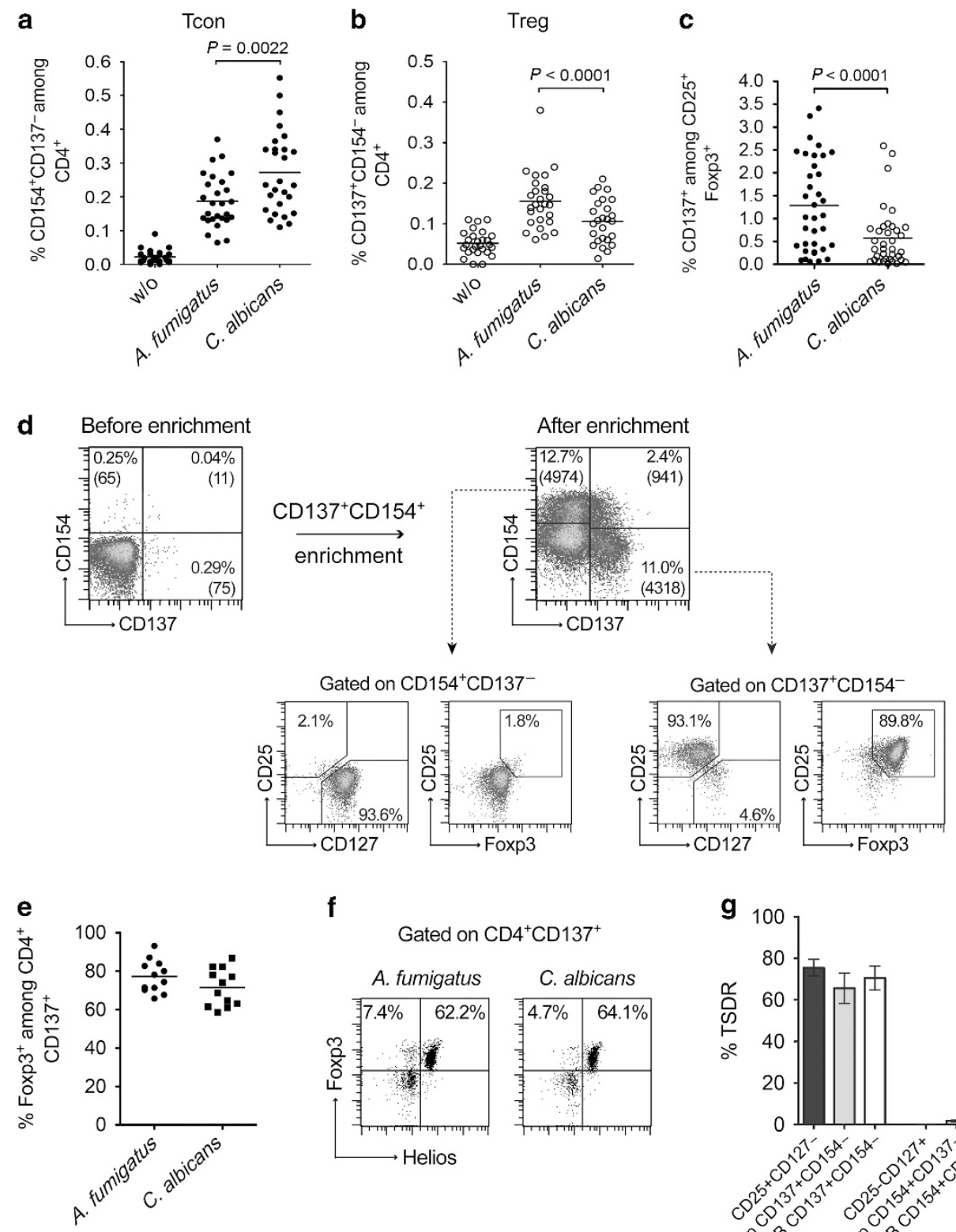

f

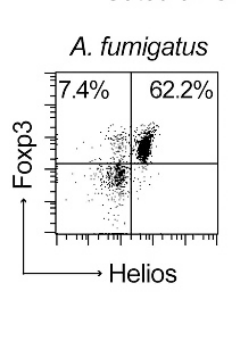

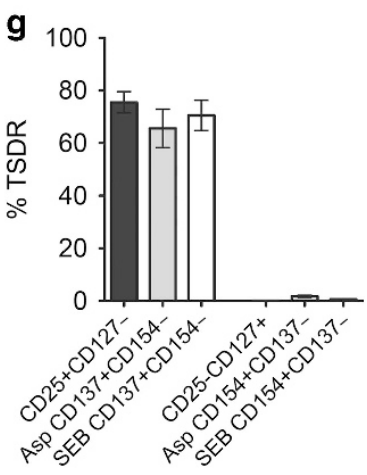

Figure 1 Combined analysis of CD154 and CD137 expression identifies in parallel fungus-specific Tcon and Treg. (a, b) PBMCs were stimulated with the indicated antigens and analyzed for CD154 and CD137 expression. For an optimized quantification of activation marker positive events among $\mathrm{CD}^{+}{ }^{+} \mathrm{CD} 4{ }^{+} \mathrm{T}$ cells, cell aggregates (scatter area vs. scatter height), dead cells and non-target cells $\left(\mathrm{CD} 14^{+}, \mathrm{CD}^{2} \mathrm{O}^{+}, \mathrm{CD} 8^{+}\right.$, dump) were excluded, and cells were counterstained for the activation marker CD69. Summary of several donors is shown with horizontal lines indicating mean values.

(c) Frequencies of reactive $\mathrm{CD} 137^{+}$cells among $\mathrm{CD} 25^{+} \mathrm{Foxp} 3^{+} \mathrm{CD} 4^{+} \mathrm{T}$ cells in PBMC. Panels a-c show the summary from seven independent experiments with $n=27$ donors. Statistical significance was determined by two-tailed paired Student's $t$-test. (d) Expressions of CD154 and CD137 on CD4 ${ }^{+} \mathrm{T}$ cells following short-term stimulation of PBMC with A. fumigatus lysate before enrichment and after combined CD154 ${ }^{+} / \mathrm{CD} 137^{+}$enrichment. Analysis of CD25, CD127 and Foxp3 expression among enriched CD154 ${ }^{+}$CD $137^{-}$CD4 ${ }^{+}$Tcon vs. CD137 ${ }^{+}$CD154 ${ }^{-}$CD4 ${ }^{+}$Treg was performed. (e) Percentage of Foxp3 expression among enriched $\mathrm{CD} 137^{+} \mathrm{CD} 4{ }^{+}$cells in several donors, following short-term stimulation with the fungal lysates. Data from three independent experiments are shown $(n=12)$. (f) Representative dot plot examples for Helios expression in enriched $\mathrm{CD} 137^{+} \mathrm{CD} 4^{+}$cells. Percentages among $\mathrm{CD} 137^{+} \mathrm{CD} 4^{+}$are indicated. Six independent experiments with several donors were performed.

(g) Polyclonal Treg and Tcon as well as A. fumigatus or SEB stimulated CD137 ${ }^{+} \mathrm{CD} 154^{-}$or $\mathrm{CD} 154^{+} \mathrm{CD} 137^{-} \mathrm{CD} 4^{+} \mathrm{T}_{\text {cells }}$ were purified by FACS and analyzed for percentage of demethylation of the Foxp3 Treg-specific demethylated region (TSDR). Graph represents mean \pm s.e.m. from five different donors. Two independent sorting experiments were performed.

of A. fumigatus-specific Treg in peripheral blood reported here.

We further analyzed whether the expanded Treg exert antigen-specific suppressive function in vitro. When
A. fumigatus antigen was added to the co-culture of allogeneic $\mathrm{CD} 4{ }^{+}$T cells with autologous APCs, the A. fumigatus-specific Treg suppressed allo-specific $\mathrm{CD} 4^{+}$T-cell proliferation already at a 1:16 ratio, whereas polyclonal Treg exerted similar 
a

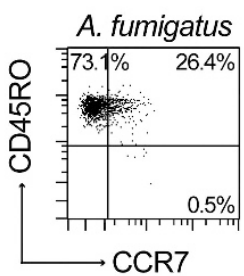

b

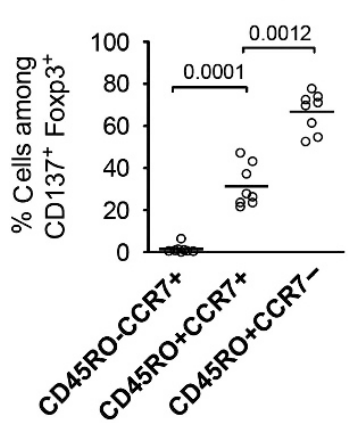

C. albicans
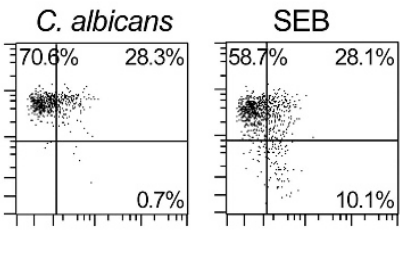

c

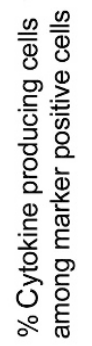

C. albicans
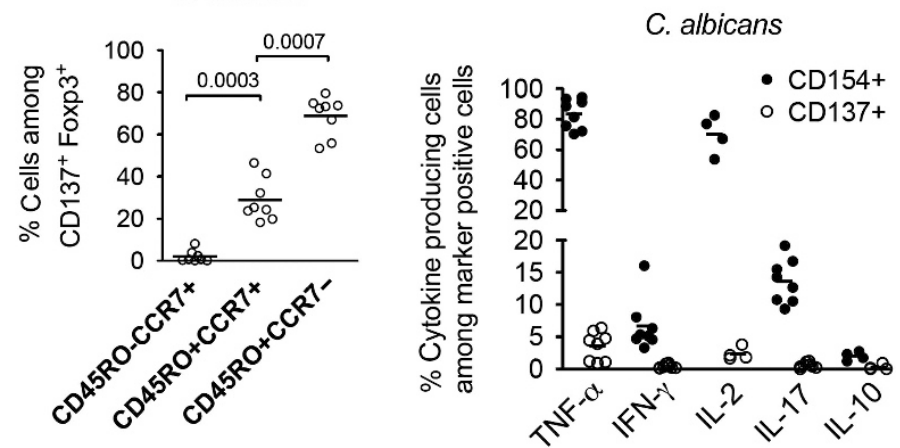

Figure 2 Fungus-specific CD137 ${ }^{+}$Treg have a memory phenotype and do not produce effector cytokines. Flow cytometric ex vivo characterization of magnetically enriched $\mathrm{CD} 154^{+}$or $\mathrm{CD} 137^{+} \mathrm{T}$ cells from short-term stimulated PBMCs. (a, b) Phenotypic characterization of fungus stimulated Treg or SEB as a high control. Enriched $\mathrm{CD} 137^{+} \mathrm{CD} 4{ }^{+} \mathrm{T}$ cells were gated on CD25 ${ }^{+} \mathrm{Foxp} 3^{+}$and analyzed for the expression of $\mathrm{CD} 45 \mathrm{RO}$ and CCR7. (a) Representative dot plot examples and (b) statistical analysis for several donors $(n=8$, two independent experiments were performed). Statistical significance was determined by two-tailed paired Student's $t$-test. (c) Fungus-specific T cells were analyzed for cytokine expression. Percentages of cytokine-expressing cells among activation marker positive CD4 ${ }^{+}$T cells are shown for several donors $(n=8$, two independent experiments were performed; for IL-2: one experiment with four different donors was performed).

suppressive activity only at a $1: 1$ ratio (Figure $3 \mathbf{b}$ and Supplementary Figure 2). As known for in vitro suppression assays, the suppressive activity of both populations was dependent on stimulation with antigen. The fact that also the weak suppressive activity of the polyclonal Treg populations is antigen-dependent again shows that polyclonal Treg contain a relatively high frequency of $A$. fumigatus-specific Treg. In contrast, both Treg populations exerted similar suppressive activity after activation with $\mathrm{CD} 3 / \mathrm{CD} 28$ beads. As a control, expanded A. fumigatus-specific CD $154^{+}$Tcon did not show comparable suppressive capacity following CD3/CD28 stimulation and only modestly inhibited when stimulated with A. fumigatus antigens, probably due to the massive expansion of the antigen-specific CD154 cells and competition for nutrients or growth factors as reported before. ${ }^{20}$ We also analyzed suppression of CD154 and TNF- $\alpha$ expressions following short-term stimulation of antigen-specific $\mathrm{T}$ cells, which is less sensitive to competitive effects due to the short stimulation time. Here A. fumigatus-specific Treg as well as polyclonal Treg, but not CD154 Tcon, showed suppressive activity (Supplementary Figure 3). Analysis of Foxp3 and Helios expressions during 3 weeks of in vitro expansion revealed that, despite the high suppressive activity, the A. fumigatus-specific Treg lost Foxp3 expression by about $50 \%$, accompanied by a loss of the demethylated TSDR region and Helios expression, in contrast to polyclonally expanded
Treg (Figure $3 \mathbf{c}$ and d). Comparable suppressive capacity and loss of Foxp3 and Helios expressions were observed for the expanded C. albicans-reactive Treg (Supplementary Figure 4).

These data demonstrate the specificity as well as the strong antigen-specific suppressive capacity of the isolated fungusspecific $\mathrm{CD} 137^{+}$Treg. However, the reduction in Foxp3 expression and the loss of Helios and TSDR demethylation during long-term in vitro expansion might indicate that at least a subset of the specific Treg has limited stability, although we cannot exclude selective outgrowth of contaminating Tcon as an alternative explanation.

\section{Minimal peripheral expansion of $\boldsymbol{A}$. fumigatus-specific Tcon and preservation of the naive repertoire}

Our data revealed so far a stronger potential of $A$. fumigatus to induce or expand antigen-specific Treg, whereas the potential to induce antigen-specific Tcon was diminished compared with C. albicans. To further explore potential differences between A. fumigatus and C. albicans-specific Tcon, we analyzed the expression of the surface markers CD45RO and CCR7 on CD $154^{+}$cells to distinguish between naive and memory T cells. As expected for peripherally expanded T cells, C. albicansreactive Tcon mainly displayed a $\mathrm{CD}_{45 \mathrm{RO}}{ }^{+}$memory phenotype (Figure $\mathbf{4 a}$ and $\mathbf{b}$ ) and were found preferentially within the $\mathrm{CCR7}^{+}$central memory compartment. For 

A. fumigatus-specific Tregs

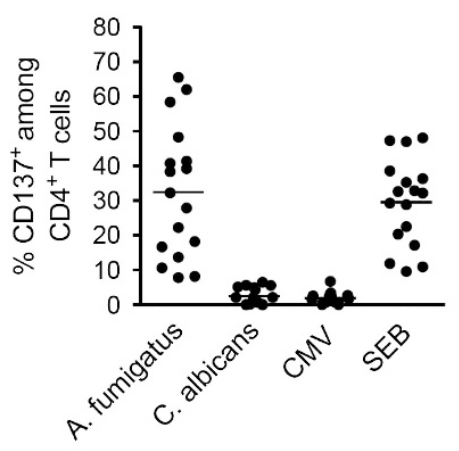

Restimulation

C. albicans-specific Tregs
Restimulation

polyclonal Tregs

b
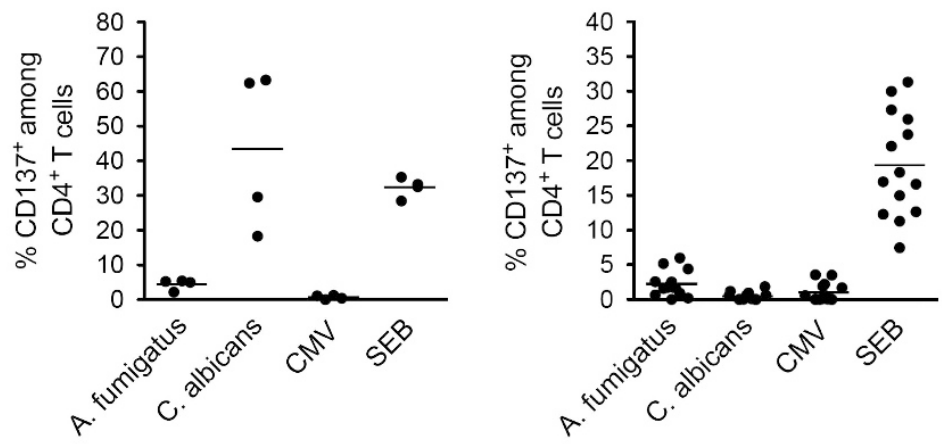

¥Specific Tregs $\triangle$ Polyclonal Tregs $\odot$ Specific CD154 T-cell lines

A. fumigatus stimulation
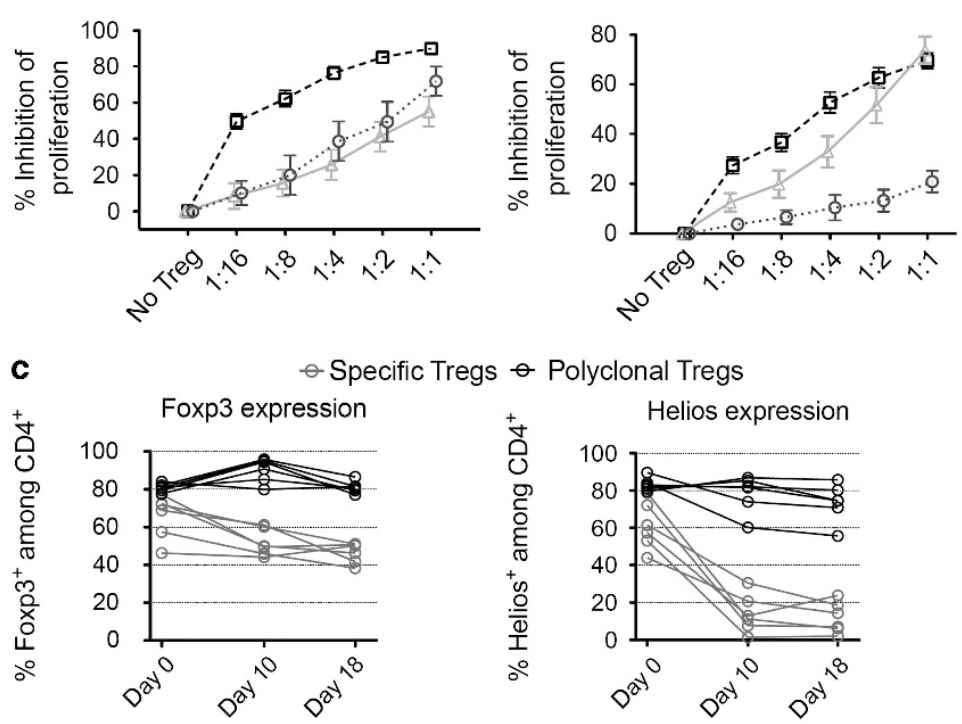

CD3/CD28 stimulation

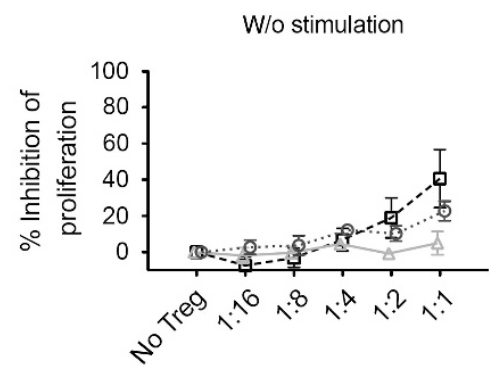

d

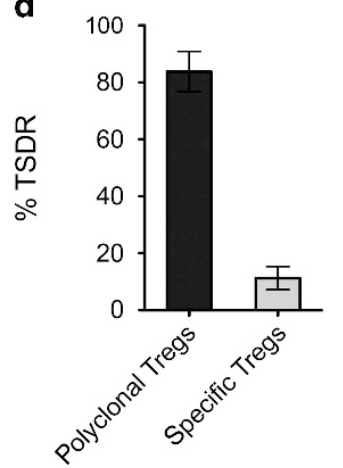

Figure 3 Specificity and suppressive capacity of fungus-specific Treg. Fungal lysate-stimulated CD137 ${ }^{+}$Treg or polyclonal CD25 $^{+} \mathrm{CD} 127^{\text {low }}$ Treg were magnetically isolated and subsequently expanded with IL-2 and rapamycin. (a) Expanded Treg were restimulated after 3 weeks of expansion in presence of autologous APCs with and without antigens as indicated and analyzed for re-expression of CD137. Statistical analysis of several donors with percentage of $\mathrm{CD} 137^{+}$cells among CD4 ${ }^{+}$lymphocytes is shown ( $A$. fumigatus Treg: $n=17$, four independent experiments were performed; C. albicans Treg: $n=4$, one experiment was performed; polyclonal Treg: $n=14$, three independent experiments were performed). (b) Expanded A. fumigatus-specific CD137 ${ }^{+}$Treg lines, A. fumigatus-specific CD154 ${ }^{+}$Tcon lines or polyclonal Treg lines were combined with autologous APCs and proliferation dye labeled allogeneic responder CD4 ${ }^{+}$T cells (Tresp) in different Treg to Tresp ratios. Percentage of inhibition of Tresp proliferation is shown without stimulation or after specific stimulation with $A$. fumigatus lysate or polyclonal stimulation with CD3/CD28. Graph represents mean \pm s.e.m. for specific and polyclonal Treg: $n=11$, three independent experiments were performed; specific CD154 ${ }^{+}$Tcon: $n=6$, two independent experiments were performed. (c) Percentage of Foxp3 and Helios expression among CD4 ${ }^{+} \mathrm{T}$ cells was analyzed by flow cytometry during expansion of $A$. fumigatus-specific CD137 ${ }^{+}$Treg or polyclonal CD25 ${ }^{+} \mathrm{CD} 127^{\mathrm{dim}}$ Treg. Representative data from two independent experiments are shown, $n=6$. (d) Demethylation analysis of the Foxp3 Treg-specific demethylated region (TSDR) in expanded A. fumigatus-specific Treg or polyclonal $\mathrm{CD} 5^{+} \mathrm{CD} 127^{\mathrm{dim}}$ Treg. Graph represents mean \pm s.e.m. from 12 different donors. Four independent expansion experiments were performed.

A. fumigatus-specific Tcon, the distribution between the two memory subsets was variable between different donors. Most interestingly, however, $20-70 \%$ of all A. fumigatus-reactive Tcon displayed a naive $\mathrm{CD}^{2} 5 \mathrm{RO}^{-} \mathrm{CCR} 7^{+}$phenotype. Further characterization indicated that these cells represent genuine naive $\mathrm{T}$ cells as demonstrated by their expression of CD45RA, CD27, a mixed phenotype for CD31, ${ }^{21}$ and lack of CD95 and
CD11a expression. Furthermore, they did not secrete effector cytokines like IFN- $\gamma$ or IL-17 (Supplementary Figure 5). The specificity of the fungus-specific $\mathrm{CD} 154^{+}$ $\mathrm{T}$ cells within the naive and memory compartment was confirmed by specific restimulation of expanded $\mathrm{CD} 154^{+}$cells from highly purified naive and memory $\mathrm{CD} 4{ }^{+} \mathrm{T}$ cells (Supplementary Figure 6). This shows that, despite chronic 

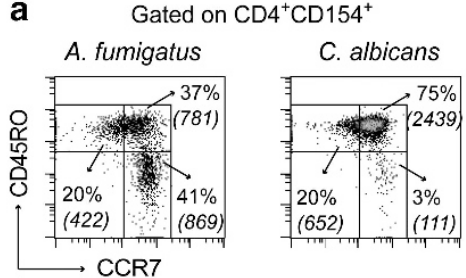
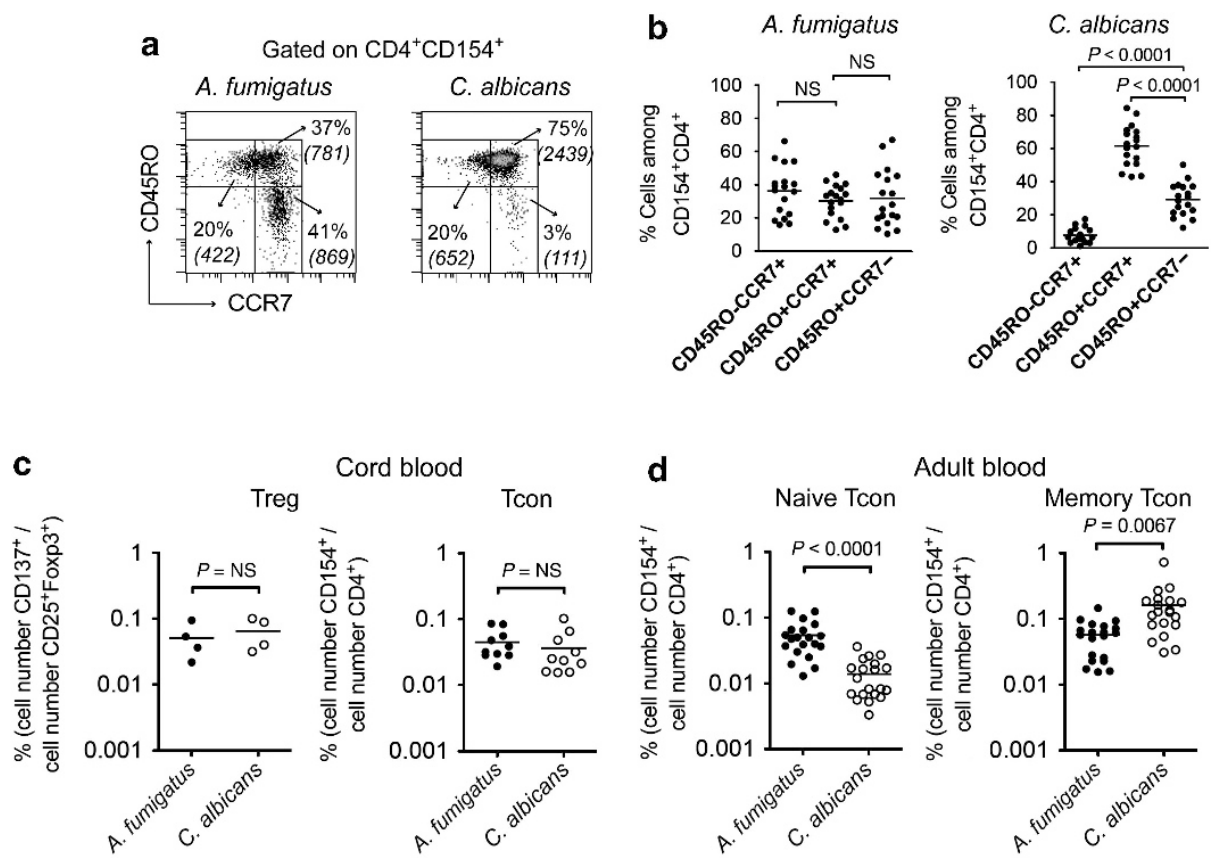

Figure 4 A high proportion of $A$. fumigatus-reactive Tcon is in a naive state. (a, b) Ex vivo analysis of CD45RO and CCR7 expression on magnetically enriched CD154 ${ }^{+} \mathrm{CD} 4{ }^{+}$Tcon. (a) Representative dot plot examples of enriched cells from $1 \times 10^{7}$ PBMCs. Percentages of cells among CD154 ${ }^{+}$and cell counts (in brackets) are indicated. (b) Phenotypic characterization of reactive CD154 ${ }^{+} \mathrm{CD} 4^{+}$Tcon in several donors. Percentages of cells among $\mathrm{CD} 154^{+} \mathrm{CD}^{+}$are indicated. Data from five independent experiments are shown, $n=18$. (c) Enumeration of fungus-reactive Treg and Tcon in cord blood. Frequencies of reactive CD137 ${ }^{+}$cells among $\mathrm{CD} 25^{+}{ }^{\mathrm{Foxp}} 3^{+} \mathrm{CD} 4^{+}$T cells $\left(n=4\right.$, four independent experiments were performed) and $\mathrm{CD} 154^{+}$ cells among CD4 ${ }^{+}$T cells ( $n=10$, six independent experiments were performed). (d) Enumeration of fungus-reactive naive and memory Tcon in adult blood. Frequencies of reactive $\mathrm{CD} 154^{+} \mathrm{CD}_{45 \mathrm{RO}}{ }^{-} \mathrm{CCR} 7^{+}$(naive) and $\mathrm{CD} 154^{+} \mathrm{CD} 45 \mathrm{RO}{ }^{+}$(memory) cells among CD4 ${ }^{+} \mathrm{T}$ cells are indicated. Data from five independent experiments are shown, $n=20$. Frequencies as in panels $\mathbf{c}$ and $\mathbf{d}$ were calculated from the total number of $\mathrm{CD} 154^{+} \mathrm{CD} 4^{+}$ or $\mathrm{CD} 137^{+} \mathrm{CD} 4^{+}$cells obtained after enrichment normalized to the total number of $\mathrm{CD} 4{ }^{+}$cells or $\mathrm{CD} 25^{+}$Foxp3 ${ }^{+} \mathrm{CD} 4^{+}$cells applied on the column. Background enriched from the non-stimulated control was subtracted. Statistical significance was determined by two-tailed paired Student's $t$-test.

exposure to A. fumigatus, large parts of the Tcon repertoire are left untouched in the naive state.

To corroborate that the enlarged memory Treg and Tcon populations in adult blood are indeed expanded by antigen contact in the periphery, we next analyzed their frequencies in cord blood. As shown in Figure 4c, the frequencies of fungusspecific Treg in cord blood were well below $0.1 \%$, corresponding to only $0.002-0.01 \%$ within the $\mathrm{CD} 4{ }^{+} \mathrm{T}$-cell population (data not shown). This demonstrates indeed a strong peripheral expansion or induction of fungus-specific Treg during adult life. Interestingly, the Tcon as well as Treg frequencies in cord blood were comparable for both fungi, indicating similar amounts of specific precursors in both repertoires (Figure 4c). However, the Tcon frequencies against A. fumigatus in cord blood were almost identical to those in adult blood (Figure 4d). This observation is consistent with the large fraction of naive Tcon in adult blood, and in summary indicates minimal peripheral expansion of $A$. fumigatus-specific Tcon. In contrast, the C. albicans-specific Tcon displayed all features of classical memory formation as the memory pool was expanded 5- to 10-fold in adult vs. cord blood, whereas the naive Tcon were reduced by a similar factor (Figure $4 \mathbf{d}$ ).

Taken together, these data confirm that both fungi, but in particular A. fumigatus, have the capacity to strongly induce or expand specific memory Treg in the periphery. However, whereas C. albicans also induces a Tmem response, the establishment of a Tmem against A. fumigatus seems to be limited, pointing toward a unique potential of $A$. fumigatus to circumvent and/or control Tmem responses via selective induction of Treg.

\section{A. fumigatus-specific Treg outnumber and functionally suppress Tmem}

To predict the actual impact of expanded Treg on the fungusspecific $\mathrm{T}$-cell response, we determined the absolute numbers of fungus-specific Treg vs. naive and memory Tcon in adult blood. Although the total Treg compartment represents only $5-10 \%$ of the $\mathrm{CD}^{+}{ }^{+} \mathrm{T}$ cells, the absolute numbers of A. fumigatusspecific Treg were equal and for some donors even exceeded the absolute number of naive or memory Tcon (Figure 5a). The dominance of the A. fumigatus Treg is further exemplified by calculating the ratio of the absolute numbers of Treg vs. Tmem for each individual donor. As shown in Figure $\mathbf{5 b}$, the A. fumigatus-specific Treg indeed exceeded Tmem by a mean factor of 1.5 (range 0.15-4.2). In sharp contrast, the C. albicansspecific T-cell pool was clearly dominated by Tmem, resulting in a reversed Treg/Tmem ratio (mean 0.38, range 0.03-1). Thus, A. fumigatus-specific Treg are strongly expanded in the periphery and can even represent the dominant population within the overall repertoire of $A$. fumigatus-specific memory 

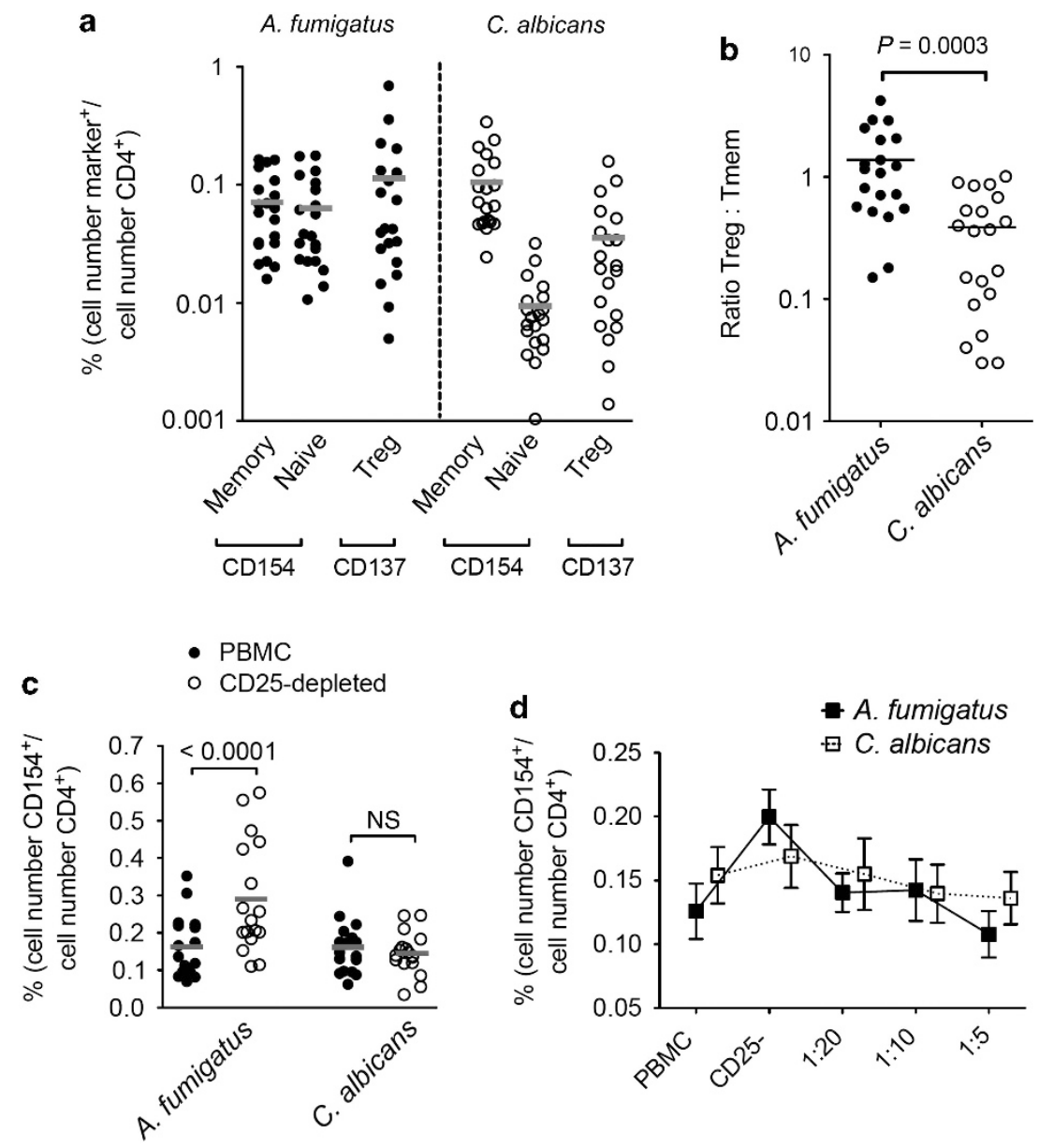

Figure 5 Reversed Treg/Tmem ratios of $A$. fumigatus- and C. albicans-specific T-cell responses. (a) A total of $1 \times 10^{7}$ PBMCs was stimulated with $A$. fumigatus or $C$. albicans and the numbers of $\mathrm{CD} 154^{+} \mathrm{CD} 137^{-}$naive and memory Tcon or CD137 ${ }^{+} \mathrm{CD} 154^{-}$Treg following combined $\mathrm{CD}_{154^{+}} / \mathrm{CD} 137^{+}$enrichment were analyzed. Frequencies were calculated from the total number of CD154 ${ }^{+}$or $^{\mathrm{CD} 137^{+}}$cells obtained after enrichment, normalized to the total number of $\mathrm{CD} 4^{+}$cells applied on the column. Background enriched from the non-stimulated control was subtracted. (b) The ratio of antigen-specific $\mathrm{CD} 137^{+} \mathrm{CD} 154^{-}$Treg to $\mathrm{CD} 154^{+} \mathrm{CD} 45 \mathrm{RO} \mathrm{O}^{+}$Tmem cells is shown. Panels a and $\mathbf{b}$ show data from five independent experiments, $n=20$. (c) Frequencies of antigen-reactive CD154 ${ }^{+}$Tcon were determined as described in panel a, in total PBMCs or after pre-depletion of CD25 ${ }^{\text {high }}$ cells $\left(n=17\right.$, five independent experiments were performed). (d) CD25 ${ }^{\text {high }}$ cells were added back to CD25 pre-depleted PBMCs with the indicated Treg to Tcon ratios. Data show mean \pm s.e.m. for six donors; two independent experiments were performed. Statistical significance was determined by two-tailed paired Student's $t$-test.

$\mathrm{CD}^{+} \mathrm{T}$ cells, whereas C. albicans induces mainly Tmem responses with minor contribution of Treg.

To confirm these data on a functional level, we analyzed the contribution of Treg to the anti-fungal T-cell response by depleting CD25 $5^{\text {high }}$ cells from PBMCs before antigen stimulation. In line with the idea that Treg control mainly the response against $A$. fumigatus, we found a significant increase in A. fumigatus-specific Tcon frequencies, whereas the frequency of C. albicans-reactive cells was not significantly altered (Figure 5c). In addition, when $\mathrm{CD} 25^{\text {high }} \mathrm{CD} 4{ }^{+} \mathrm{T}$ cells were added back into the CD25-depleted fraction, the frequency of A. fumigatus-specific Tcon dropped to the original level (Figure 5d).

Taken together, these results demonstrate that $A$. fumigatus triggers the selective expansion of an antigen-specific Treg pool in healthy human donors, which suppresses Tcon activation in vitro, and thus may contribute to tolerance by limiting the formation of an expanded Tmem pool.

\section{Cystic fibrosis patients with Aspergillus-associated allergies are characterized by a shift of the A. fumigatus-specific Treg/Tmem ratio}

To identify a potential role of $A$. fumigatus-specific Treg in mediation of tolerance in vivo, we determined whether this protective Treg/Tmem ratio is altered under pathological conditions. To this end we analyzed samples from patients with CF (Supplementary Table 1 online), which are known to frequently suffer from lung infections and severe allergic reactions against $A$. fumigatus. We were able to identify CF patients with severe allergic reactions against $A$. fumigatus based on a strong Th2 effector cytokine expression of A. fumigatusreactive $\mathrm{CD} 154^{+}$Tcon (i.e. production of IL-13, IL-4 and IL-5) (Figure 6a and b), which also corresponded with Aspergillusspecific IgE levels (Figure 6c). By contrast, in non-Th2 CF patients the cytokine profiles of $\mathrm{CD} 154^{+}$A. fumigatus-specific Tcon did not differ from those of healthy donors, i.e., they produced mainly TNF- $\alpha$ and IFN- $\gamma$ but no Th2 cytokines. Strikingly, 
a

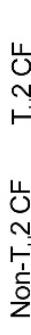

Gated on $\mathrm{CD} 4^{+} \mathrm{CD} 154^{+}$
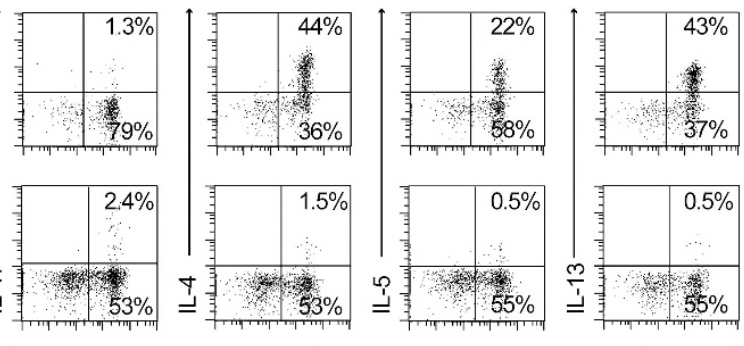

CD45RO

b

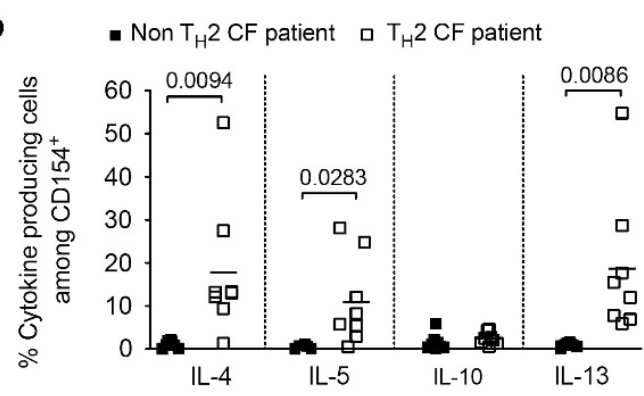

- Non $\mathrm{T}_{\mathrm{H}} 2$ CF patient $\square \mathrm{T}_{\mathrm{H}^{2}}$ CF patient
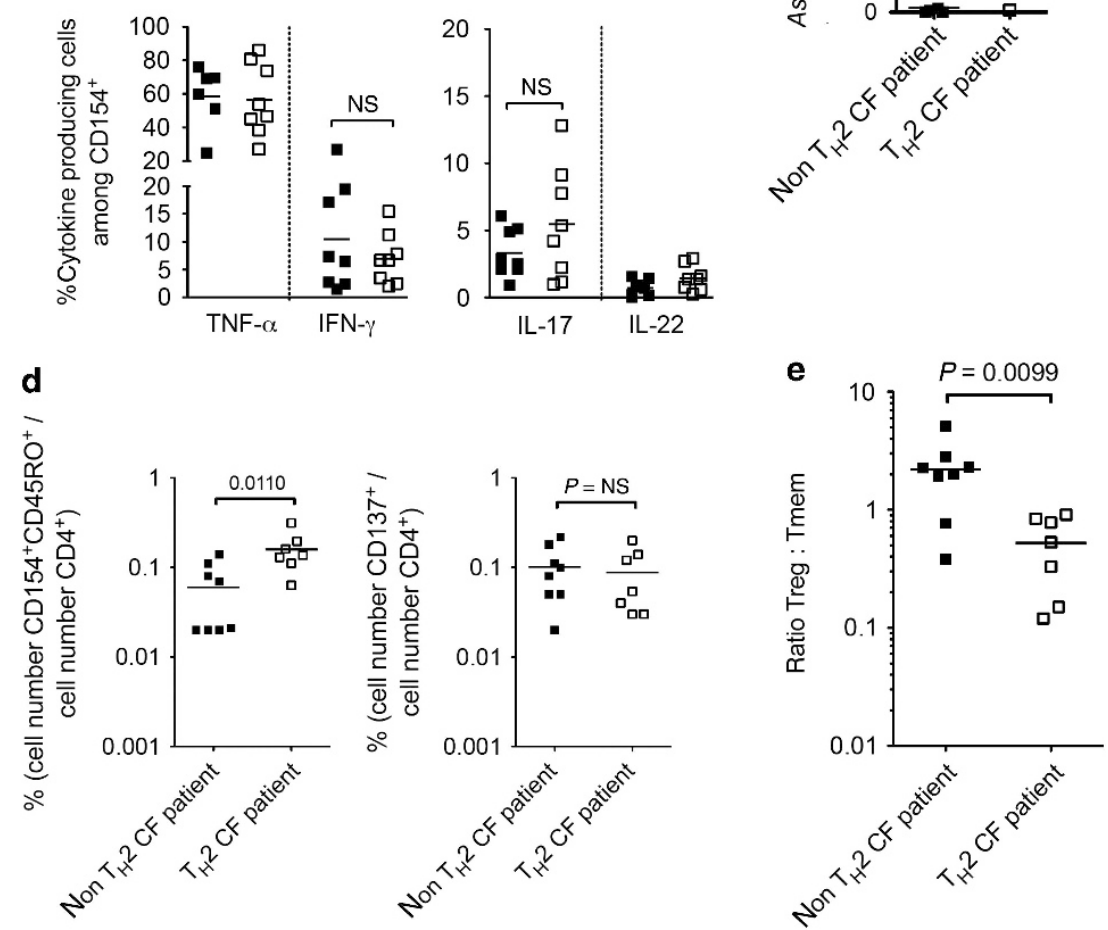

Figure 6 Frequencies of $A$. fumigatus-specific Tmem and Treg in peripheral blood of cystic fibrosis patients. (a, b) PBMCs from CF patients were stimulated with $A$. fumigatus lysate. Reactive CD154 ${ }^{+}$Tcon were magnetically enriched and ex vivo analyzed for cytokine expression. CF patients were classified according to $A$. fumigatus-specific cytokine expression in Th2 and non-Th2 patients using a cutoff value of $>5 \% \mathrm{IL}-13$ producing $A$. fumigatusreactive CD154 ${ }^{+}$T cells. (a) Representative dot plot examples and (b) statistical analysis with percentages of cytokine-expressing cells among CD154 ${ }^{+} \mathrm{CD}^{+}$are shown. (c) A. fumigatus-specific serum IgE levels. (d) Frequencies of CD154 ${ }^{+} \mathrm{CD}_{5} \mathrm{RO}^{+} \mathrm{Tmem}$ among CD4 ${ }^{+} \mathrm{T}$ cells and A. fumigatus-reactive $\mathrm{CD} 137^{+} \mathrm{CD} 154^{-}$Treg among CD4 ${ }^{+} \mathrm{T}$ cells. (e) Ratio of $A$. fumigatus-specific Treg to Tmem. Panels $\mathbf{b}-\mathbf{e}$ show a summary from eight non-Th2 CF patients and eight Th2 CF patients; 13 independent experiments were performed. Statistical significance was determined using two-tailed unpaired Student's $t$-test.

comparing the frequencies of Treg and Tmem cells in both patient groups yielded a two- to fourfold increase of A. fumigatus-specific Tmem in Th2 CF patients vs. non-Th2 patients and healthy controls (Figure 6d and data not shown). However, fungusspecific Treg frequencies were similar in both patients groups
(Figure 6d). Thus, the overall ratio of A. fumigatus-specific Treg to Tmem was completely reversed in the Th2 CF patient group toward a dominance of Tmem (mean Treg/Tmem 0.52, range 0.12-0.91) (Figure 6e). These data highlight that the physiological dominance of specific Treg vs. Tmem in healthy 
subjects probably represents an important mechanism to prevent allergy induction.

\section{DISCUSSION}

Humans are continuously challenged by various pathogenic fungi, which are either part of the commensal microbiota like C. albicans or present in the environment, e.g., in the air that we breath, like A. fumigatus. This requires balanced pathogenspecific control mechanisms, which allow effective protection but prevent collateral tissue damage caused by chronic inflammation or allergy. How the human immune system achieves this balance for the various fungal species is currently not well understood. We addressed this question by applying a parallel quantitative assessment of human fungus-specific $\mathrm{CD}^{+} \mathrm{T}$ cells within the naive, memory and Treg repertoire. This approach revealed that both fungi generate unexpectedly high frequencies of specific Treg. Surprisingly, for A. fumigatus this was not accompanied by a parallel increase in Tmem, and thus resulted in a Treg dominance characterized by a high Treg/ Tmem ratio (mean 1.5), as well as preservation of a large naive Tcon population and significant suppression of A. fumigatusspecific T-cell activation in vitro. The protective Treg/Tmem balance was completely reversed in CF patients with allergic reactions against $A$. fumigatus due to the formation of a strong Th2-type Tmem response. To the best of our knowledge, this is the first direct evidence for the formation of a preferential antigen-specific Treg vs. Tmem response in humans, representing a potential mechanism for the protection from Th2associated immunopathology against an opportunistic environmental pathogen.

In the majority of donors analyzed, A. fumigatus-specific Treg even outnumber specific Tmem. In addition, the specific naive T-cell pool is largely preserved in size when compared with cord blood, suggesting a limited Tcon response against A. fumigatus antigens. In contrast, the C. albicans-reactive T-cell repertoire mainly consisted of Tmem, which exceeded A. fumigatus-specific Tmem by a factor of $\sim 2-5$. At the same time, a depletion from the naive pool was observed as it has been reported for other pathogen-specific T-cell responses. ${ }^{22}$ Although we clearly detected specific Treg against C. albicans, they occurred at lower frequencies than specific Tmem (mean ratio Treg/Tmem 0.38) and A. fumigatus-specific Treg. In this respect, the T-cell response against $C$. albicans is similar to several other anti-pathogen $\mathrm{T}$-cell responses, including viruses, parasites and bacteria, where antigen-specific Treg expansion occurs along with a strong effector T-cell response. ${ }^{23-25}$ The dominant role of the enlarged A. fumigatus- but not C. albicans-specific Treg pool is further supported by functional data showing that total Treg depletion results in an increased stimulation of A. fumigatus but not C. albicansspecific Tcon.

The data reported here clearly demonstrate a fundamental difference between the T-cell responses against $A$. fumigatus and $C$. albicans beyond the previously described differences with regard to effector cytokine production by Tmem. ${ }^{18,26-29}$ Whereas C. albicans generates a classical Th17-dominated anti- pathogen T-cell response with only a minor contribution of specific Treg, A. fumigatus seems to preferentially expand Treg and rather preserves the naive $\mathrm{T}$-cell repertoire. This difference may be due to the nature of the pathogen, e.g., expression of pathogen-associated molecular patterns, the infection route or the antigen dose. As A. fumigatus is a ubiquitously distributed airborne fungus, humans acquire it primarily by inhaling spores. In contrast, the commensal C. albicans is mainly residing on the mucosal surface of the gastrointestinal tract and skin. However, both the gut and the lung are known as preferential sites for the generation of Treg responses against commensals and environmental antigens. ${ }^{2,5,6,30-33}$ As for now, there is no evidence that A. fumigatus has an inherent tolerogenic potential but rather has been reported to generate effector T-cell responses after repeated nasal exposure with A. fumigatus conidia in vivo. ${ }^{34}$ Therefore, it seems likely that antigen dosing and timing are relevant parameters for the preferential Treg activation by A. fumigatus antigens.

C. albicans invasion usually occurs in a spatially and timely restricted manner, e.g., after epithelial damage, ${ }^{35}$ which probably results in a transiently high local antigen concentration and presumably leads to a strong immune reaction. In contrast, the continuous inhalation of airborne A. fumigatus spores may rather favor the disseminated and chronic presence of antigens at low concentrations. Furthermore, innate immune recognition of dormant $A$. fumigatus spores is largely prevented by a hydrophobin surface layer, ${ }^{36,37}$ which may further diminish antigen uptake and presentation. The chronic exposure to low doses of antigens has previously been described to support the generation of Treg responses. ${ }^{38-40}$ In addition, the capacity of lung macrophages to induce Treg from naive T cells has recently been reported for the murine system. ${ }^{41}$ This further supports the observed preferential Treg response against inhaled A. fumigatus antigens.

Because of the lack of distinctive markers in humans, the origin of the fungus-specific Treg remains speculative. The analysis of cord blood samples clearly showed that A. fumigatus-specific, as well as C. albicans-specific, Treg and Tcon are present in similar but very low frequencies at birth, proving that the enlarged Treg pool is the result of an antigen-specific induction or expansion process during life. This is also in accordance with the $\mathrm{CD} 45 \mathrm{RO}^{+}$memory phenotype of the fungus-specific Treg. However, the question whether the specific Treg populations arise from expanded thymic (tTreg) or peripherally induced Treg (pTreg) remains. Our ex vivo phenotypic analysis revealed hallmarks of tTreg, i.e., fully demethylated TSDR, expression of Helios, lack of cytokine and CD127 expression. A thymic origin would also be in accordance with our finding that in adult blood the naive Tcon pool was not significantly depleted of A. fumigatusreactive cells. Moreover, tTreg have recently been shown to significantly contribute to the T-cell repertoire specific for intestinal commensal microbiota. ${ }^{2}$ In contrast, pTreg formation against airborne antigens seems to be essential for the maintenance of tolerance at mucosal sites, as mice specifically lacking $\mathrm{p}$ Treg selectively develop pathological Th2 responses at 
mucosal surfaces. ${ }^{5}$ This leaves open the possibility that the fungus-specific Treg population is actually composed of pTreg as well as expanded tTreg. This could also explain why Helios and Foxp3 expressions, as well as TSDR demethylation, were lost at least by a subset of fungus-reactive Treg upon prolonged in vitro expansion. Such phenotype instability has been described for in vitro-induced $\mathrm{Treg}^{42}$ and long-term cultured $\mathrm{CD} 25^{+} \mathrm{CD} 127^{-} \mathrm{CD} 45 \mathrm{RO}^{+}$memory Treg. ${ }^{43}$ However, selective outgrowth of effector $\mathrm{T}$ cells even in the presence of rapamycin cannot be excluded.

Our study has also important clinical implications, as A. fumigatus is the cause of several diseases. In immunocompetent individuals repeated exposure to A. fumigatus can trigger pathological Th2 responses associated with strong allergic reactions such as asthma or ABPA. Especially, CF patients frequently suffer from Aspergillus colonization of the lung and associated allergic responses. ${ }^{44}$ However, the reasons why some patients develop a severe pathologic Th2 response while others do not are currently unknown. Intriguingly, we observed that those CF patients with a strong Th2 bias of the A. fumigatus-specific Tcon, which also corresponded with increased A. fumigatus-specific IgE levels, displayed a marked shift of the Treg/Tmem ratio (from 2.1 to 0.5 ). This shift was due to the selective expansion of A. fumigatus Th2 Tmem, whereas Treg numbers remained constant, suggesting no direct defect in Treg generation in CF patients. There is also no other evidence that Treg function may generally be affected by the CFTR gene mutations underlying cystic fibrosis. Thus, our data suggest that A. fumigatus-specific Th2 differentiation and expansion can be initiated by escaping Treg control under the specific conditions of CF lung environment. This escape is most likely related to the increased $A$. fumigatus load $^{44}$ due to defective epithelia or phagocyte function, ${ }^{45}$ which may lead to alterations in antigen density and APC composition or activation status. In any case, these data provide direct evidence for the hypothesis that Treg formation is an important process to protect from allergy development against chronically inhaled A. fumigatus antigens in healthy subjects and that alterations in this process or an imbalance between pathologic Tmem vs. Treg contribute to disease development or chronification, as demonstrated in the murine system. ${ }^{5,46-48}$ In line with this, patients with immune dysregulation, polyendocrinopathy, enteropathy, X-linked (IPEX) syndrome, who are deficient for Treg, have indeed elevated levels of IgE, and recently a case of ABPA in an IPEX patient occurring at an unusually young age was reported. ${ }^{49}$ In contrast, immunodeficient patients are prone to contracting life-threatening invasive Aspergillus infections with high mortality rates $(50-90 \%) .{ }^{8}$ For this patient group, the capacity of A. fumigatus to preferentially generate a Treg response may even represent a potential risk factor, as it prevents or impairs protective effector T-cell responses. Indeed, increased levels of the immunosuppressive cytokine IL-10 have been associated with a poor disease outcome in patients with invasive aspergillosis, ${ }^{50}$ although there is so far no direct evidence for the presence of $A$. fumigatus-specific Treg in these patients.
Beyond the immediate relevance of our findings for human diseases related to A. fumigatus infections, delineating the mechanisms how A. fumigatus induces selective Treg vs. Tcon responses in humans will help to design therapeutic strategies aiming at the induction of antigen-specific Treg.

\section{METHODS}

Blood donors. Buffy coats or peripheral ethylenediamine tetraacetic acid (EDTA) blood samples from healthy human donors were obtained from the Institute for Transfusion Medicine, University Hospital Dortmund, Germany and from in-house volunteers. Peripheral blood samples from 16 patients with a diagnosis of cystic fibrosis (CF) were obtained from the University Hospital Cologne, Germany. Blood sampling was performed within the scope of the biomaterial repository protocol ISI, and all patients gave informed consent (local ethics identifier 08-160). CF patients were classified according to the A. fumigatus-stimulated production of cytokines. A cutoff value of $>5 \%$ IL-13 producing A. fumigatus-reactive $\mathrm{CD} 154^{+} \mathrm{T}$ cells was determined to discriminate between Th2 and non-Th2 patients.

Cell preparation and stimulation. Peripheral blood mononuclear cells (PBMCs) were isolated by Ficoll-Paque (GE Healthcare Life Sciences, Freiburg, Germany) density gradient centrifugation and resuspended in RPMI-1640 (Miltenyi Biotec, Bergisch Gladbach, Germany), supplemented with $5 \%(\mathrm{v} / \mathrm{v})$ human AB-serum (BioWhittaker/Lonza, Walkersville, MD), 2 mM L-glutamine (PAA Laboratories, Pasching, Austria). Cells were stimulated with the following antigens: A. fumigatus lysate $\left(40 \mu \mathrm{g} \mathrm{ml}^{-1}\right.$; generated as described $),{ }^{18}$ C. albicans-lysate $\left(40 \mu \mathrm{g} \mathrm{ml}^{-1}\right.$; Greer Laboratories, Lenoir, NC), CMV-lysate $\left(10 \mu \mathrm{g} \mathrm{ml}^{-1}\right.$, Siemens Healthcare Diagnostics, Marburg, Germany), Staphylococcal enterotoxin B (SEB $1 \mu \mathrm{g} \mathrm{ml}^{-1}$, Sigma Aldrich, Schnelldorf, Germany) in presence of $1 \mu \mathrm{g} \mathrm{ml}^{-1} \mathrm{CD} 40$, $1 \mu \mathrm{g} \mathrm{ml}^{-1} \mathrm{CD} 28$ pure antibodies (both Miltenyi Biotec).

Flow cytometry. Cells were stained in different combinations with the following monoclonal antibodies according to manufacturers' protocols: CD4-VioBlue, CD4-FITC, CD4-PE-Vio770, CD4APC-Vio770 (VIT4), CD3-VioBlue (BW264/56), CD8-VioGreen, CD8-PerCP (BW135/80), CD14-VioGreen, CD14-PerCP (TÜK4), CD20-VioGreen, CD20-PerCP (LT20), CD69-FITC (FN50), CD137PE, CD137-APC (4B4-1), CD154-VioBlue, CD154-FITC, CD154APC (5C8), CD25-PE, CD25-APC (4E3), CD127-FITC (MB15-18C9), CD45RO-PE-Vio770, CD45RO-APC (UCHL1), TNF- $\alpha$-FITC, TNF- $\alpha$-PE (cA2), IFN- $\gamma$-APC (45-15), IL-17A-APC, IL-17A-FITC (CZ8-23G1), IL-2-APC (N7.48A), IL-10-APC (JES3-9D7), IL-4-PE (7A3-3), IL-5-APC (JES1-39D10), IL-13-PE (JES10-5A2.2), Foxp3APC (3G3) (all from Miltenyi Biotec), Foxp3-PerCP-Cy5.5 (PCH101) IL-22-PerCP-Cy5.5 (22URTI) (both from eBioscience, San Diego, CA, USA), Helios-FITC, Helios-PE, (22F6), CCR7-Alexa Fluor 488 (G043H7), IFN- $\gamma$-PerCP-Cy5.5 (4S.B3) (all from BioLegend, San Diego, CA, USA), TNF- $\alpha$-VioBlue (cA2), IFN- $\gamma$-VioBlue (45-15), HLA-A2-PE (BB7.2) (all conjugated in-house). Data were acquired on a MACSQuant analyzer. MACSQuantify software was used for analysis (both Miltenyi Biotec).

Enrichment and characterization of antigen-reactive T cells. A total of $1 \times 10^{7}$ PBMCs per $\mathrm{ml}$ was stimulated for $7 \mathrm{~h}$ with different antigens. For combination of antigen-reactive T-cell enrichment with an intracellular staining, $1 \mu \mathrm{g} \mathrm{ml}^{-1}$ Brefeldin A (Sigma Aldrich) was added for the last $2 \mathrm{~h}$. Cells were separated using the CD154 MicroBead Kit and/or the CD137 MicroBead Kit (both Miltenyi Biotec) either alone or in combination. In brief, cells were indirectly magnetically labeled with CD154-Biotin and CD137-PE, followed by anti-Biotin and anti-PE MicroBeads and enriched by two sequential MS columns (Miltenyi Biotec). Surface staining was performed on the first column, 
followed by fixation and intracellular cytokine staining on the second column (Inside stain Kit; Miltenyi Biotec), as described, ${ }^{18}$ or staining for transcription factors using the Foxp3 Staining Buffer Set (Miltenyi Biotec). In some experiments, CD25 $5^{\text {high }}$ cells were depleted before stimulation and enrichment of antigen-reactive CD154 ${ }^{+}$T cells, using CD25 MicroBeads and LD columns (both Miltenyi Biotec).

In vitroT-cell expansion and restimulation. Stimulation and isolation of antigen-specific $\mathrm{T}$ cells was performed as described above. Polyclonal Treg were isolated with the $\mathrm{CD} 4{ }^{+} \mathrm{CD} 25^{+} \mathrm{CD} 127^{\mathrm{dim} /-}$ Regulatory T-cell Isolation Kit (Miltenyi Biotec). A total of $1 \times 10^{5}$ per well polyclonal Treg and $1 \times 10^{4}$ per well CD137 ${ }^{+}$cells were cultured in 96-well round-bottom plates in X-Vivo15 (BioWhittaker/Lonza), supplemented with $5 \%(\mathrm{v} / \mathrm{v})$ human $\mathrm{AB}$-serum, $500 \mathrm{U} \mathrm{ml}^{-1} \mathrm{IL}-2$ (Proleukin; Novartis, Nürnberg, Germany), $30 \mathrm{~nm}$ rapamycin (Sigma Aldrich), and $100 \mathrm{IU} \mathrm{ml}^{-1}$ penicillin, $100 \mathrm{\mu g} \mathrm{ml}^{-1}$ streptomycin, $0.25 \mu \mathrm{g} \mathrm{ml}^{-1}$ amphotericin B (Antibiotic Antimycotic Solution, Sigma Aldrich). For polyclonal Treg, 4:1 (bead-to-cell ratio) Treg Expansion Beads (Miltenyi Biotec) were added. Cells were expanded for 21 days, with polyclonal Treg being restimulated 2:1 (bead-to-cell ratio) at day 10 .

Isolated CD154 ${ }^{+}$cells were cultured for 14 days, at a density of $2.5 \times 10^{6}$ cells per $\mathrm{cm}^{2}$ with 1:100 mitomycin C (Sigma Aldrich) treated autologous feeder cells in X-Vivo15, supplemented with $5 \%$ $(\mathrm{v} / \mathrm{v}) \mathrm{AB}$-serum, $200 \mathrm{U} \mathrm{ml}^{-1} \mathrm{IL}-2$ and $100 \mathrm{U} \mathrm{ml}^{-1}$ penicillin, $100 \mu \mathrm{g} \mathrm{ml}^{-1}$ streptomycin, $0.25 \mu \mathrm{g} \mathrm{ml}^{-1}$ amphotericin B. During expansion medium was replenished, and cells were split as needed. For expansion of T-cell lines from the naive or memory repertoire, $\mathrm{CD} 4{ }^{+} \mathrm{T}$ cells were isolated by negative selection using the naive $\mathrm{CD} 4{ }^{+} \mathrm{T}$ cell-Isolation Kit or the memory $\mathrm{CD} 4{ }^{+} \mathrm{T}$ cell Isolation Kit (both Miltenyi Biotec), respectively. A total of $1 \times 10^{7}$ isolated naive or memory $\mathrm{CD}^{+}{ }^{+} \mathrm{T}$ cells were stimulated with autologous $\mathrm{CD} 3$-depleted PBMCs as APCs in a ratio of 1:1 and fungal lysates. Enrichment and cultivation of reactive $\mathrm{CD} 154^{+}$cells from both CD ${ }^{+}{ }^{+}$-cell subsets was performed as described above.

Before restimulation, expanded cells were rested in RPMI$1640+5 \%$ human AB-serum for 2 days. A total of $5 \times 10^{5}$ expanded cells were re-stimulated with autologous CD3-depleted PBMCs (CD3 MicroBeads, LD columns; both Miltenyi Biotec) in a ratio of $1: 1$ in 96 -well flat bottom plates in presence of $1 \mu \mathrm{g} \mathrm{ml}^{-1} \mathrm{CD} 28$ functional grade pure $\mathrm{Ab}$ for $2 \mathrm{~h}$ plus additionally $4 \mathrm{~h}$ with $1 \mu \mathrm{g} \mathrm{ml}{ }^{-1}$ Brefeldin A (Sigma Aldrich). After fixation and permeabilization, cells were stained intracellularly for CD137 and Foxp3 expression (Foxp3 Staining Buffer Set; Miltenyi Biotec).

Stimulation of cord blood samples. Cord blood samples were obtained from local hospitals after informed consent. CD14 ${ }^{+}$ monocytes were isolated from cord blood mononuclear cells (CBMCs) by positive selection with CD14 MicroBeads (Miltenyi Biotec) and cultivated for 2 days with an antigen in X-Vivo15 (BioWhittaker/ Lonza), supplemented with $1000 \mathrm{IU} \mathrm{ml}^{-1} \mathrm{GM}-\mathrm{CSF}$ and $400 \mathrm{IU} \mathrm{ml}^{-1}$ IL-4 (both Miltenyi Biotec) to generate fastDC. DCs were maturated for 1 day with $1000 \mathrm{IU} \mathrm{ml}^{-}$TNF- $\alpha, 1000 \mathrm{IU} \mathrm{ml}^{-1}$ IL1- $\beta$ (both Miltenyi Biotec), $1 \mu \mathrm{g} \mathrm{ml}^{-1} \mathrm{PGE}_{2}$ (Sigma Aldrich) and were used for stimulation of the autologous $\mathrm{CD}^{-} 4^{-}$fraction. Enrichment of $\mathrm{CD}_{154}{ }^{+}$and $\mathrm{CD} 137^{+} \mathrm{T}$ cells was performed as described above.

For restimulation of expanded CD $154^{+}$cells from cord blood samples, $\mathrm{CD}^{+}{ }^{+}$cells were isolated using the CD34 MicroBead Kit (Miltenyi Biotec) and were cultured for 3 weeks in $\mathrm{X}-\mathrm{Vivo}{ }^{\mathrm{TM}} 15$ supplemented with $5 \%$ (v/v) AB-serum, $25 \mathrm{ng} / \mathrm{ml}$ Flt3-L, $10 \mathrm{ng} / \mathrm{ml}$ TPO and $20 \mathrm{ng} / \mathrm{ml} \mathrm{SCF}$ (all Miltenyi Biotec) at a density of $1 \times 10^{5} / \mathrm{ml}$. At day $21, \mathrm{CD}_{14}{ }^{+}$cells were isolated with CD14 MicroBeads (Miltenyi Biotec) and used for restimulation of the expanded CD154 ${ }^{+} \mathrm{T}$ cells.

Proliferation assay. For proliferation assays, $\mathrm{CD} 4{ }^{+}$responder T cells (Tresp) were untouched isolated with the $\mathrm{CD} 4{ }^{+} \mathrm{T}$ cell Isolation Kit (Miltenyi Biotec) and labeled with the CellTrace Violet Cell Proliferation Kit (Invitrogen, Molecular Probes, Eugene, OR) at a final concentration of $2.5 \mu \mathrm{M}$. A total of $2 \times 10^{5}$ Tresp were co-cultured in different ratios with expanded polyclonal or specific Treg or expanded CD154 T cell lines as a control, and $4 \times 10^{5}$ Treg-autologous CD3-depleted PBMCs as APCs and inducer of an allo-reaction. Treg were either stimulated specifically with $40 \mu \mathrm{g} \mathrm{ml}^{-1}$ A. fumigatus lysate or polyclonal with anti-CD3/CD28 beads (Treg Suppression Inspector; Miltenyi Biotec). On day 6, dilution of proliferation dye was analyzed by flow cytometry. Tresp could be discriminated from Treg and APCs by using donors with opposite HLA-A2 expression ${ }^{19}$ (Supplementary Figure 2a).

Suppression of $\mathrm{CD} 154^{+}$induction. Tresp were generated by expansion of A. fumigatus-specific CD154 ${ }^{+} \mathrm{T}$ cell lines, as described above and stained with the CellTrace Violet Cell Proliferation dye (Invitrogen, Molecular Probes). A total of $2 \times 10^{5}$ Tresp were co-cultured in different ratios with expanded polyclonal or specific Treg from the same donor and $4 \times 10^{5} \mathrm{CD} 3$-depleted PBMCs as APCs. Specific T cells were stimulated with A. fumigatus lysate and polyclonal Treg with anti-CD3/CD28 beads in presence of $1 \mu \mathrm{g} \mathrm{ml}^{-1} \mathrm{CD} 28$ functional grade pure $\mathrm{Ab}$ for $2 \mathrm{~h}$ plus additionally $4 \mathrm{~h}$ with $1 \mu \mathrm{g} \mathrm{ml}^{-1}$ Brefeldin A (Sigma Aldrich) and stained intracellular for CD154 and TNF- $\alpha$ induction. Tresp and Treg could be discriminated by Violet Dye staining of the responder cells (Supplementary Figure 3a).

Methylation analysis of Foxp3 Treg-specific demethylated region (TSDR). After centrifugation, cell pellets were dissolved in $400 \mu \mathrm{l} \mathrm{PBS}$, and genomic DNA was isolated using the QIAamp ${ }^{\mathrm{R}}$ DNA Blood Mini Kit (Qiagen, Hilden, Germany). Five-hundred nanograms of eluted genomic DNA was used in a subsequent bisulfite treatment $\left(\right.$ EpiTect $^{\mathrm{R}}$, Qiagen). A minimum of $60 \mathrm{ng}$ bisulfite-treated genomic DNA was then used in a real-time-PCR to quantify the FOXP3 TSDR. Real-timePCR was performed in a final reaction volume of $20 \mu$ containing $10 \mu$ FastStart Universal Probe Master (ROX) (Roche Diagnostics, Mannheim, Germany), $50 \mathrm{ng} \mathrm{\mu l}^{-1}$ Lamda DNA (New England Biolabs, Frankfurt, Germany), $5 \mathrm{pmol} \mu \mathrm{l}^{-1}$ methylation or nonmethylation specific probe, $30 \mathrm{pmol} \mu \mathrm{l}^{-1}$ methylation or non-methylation specific primers and $60 \mathrm{ng}$ bisulfite-treated DNA or respective amount of plasmid standard. The samples were analyzed in triplicates on an ABI 7500 Cycler using the following cycling conditions: 1 cycle of $10 \mathrm{~min} 95^{\circ} \mathrm{C}$ and 45 cycles of $15 \mathrm{~s} 95^{\circ} \mathrm{C}$ followed by $1 \mathrm{~min} 61^{\circ} \mathrm{C}$. \% FOXP3 TSDR content was then calculated by dividing the nonmethylated copy-number by the total genomic FOXP3 copy-number. For detailed instruction see also. ${ }^{51}$

Statistics. Statistical analyses were performed with the GraphPad PRISM software 5.0 (GraphPad Software, La Jolla, CA). Significance was determined by two-tailed Student's $t$-test as indicated. $P$-values of $<0.05$ were considered statistically significant.

SUPPLEMENTARY MATERIAL is linked to the online version of the paper at http://www.nature.com/mi

\section{ACKNOWLEDGMENTS}

We thank Maria Pötsch (Hans-Knoell Institute Jena, Germany) for her excellent technical support and Gunter Rappl (Central Cell Sorting Facility, Center for Molecular Medicine Cologne, Germany) for FACS sorting. This research was supported by the European Union, Project "Development of Novel Management Strategies for Invasive Aspergillosis-MANASP" (contract number LSHE-CT-2006-037899) (to P.B., O.K., M.A., A.A.B., A.S.), by the European Union 7th Framework Program as part of the project Nanoll, grant agreement no.: 229289 (to P.B., M.A., A.S.) and by grants from the Deutsche Forschungsgemeinschaft, Sonderforschungsbereich 633 and Sonderforschungsbereich 650 .

\section{DISCLOSURE}

M.A. works as an employee of Miltenyi Biotec and A.S. works as a consultant for Miltenyi Biotec. O.A.C. has received research grants from $3 \mathrm{M}$, Actelion, Astellas, Basilea, Bayer, Biocryst, Celgene, Cubist, F2G, Genzyme, Gilead, GSK, Merck/MSD, Miltenyi, Optimer, Pfizer, Quintiles, 
and Viropharma, is a consultant to $3 \mathrm{M}$, Astellas, Basilea, F2G, Gilead, Merck/MSD, Optimer, and Pfizer, and received lecture honoraria from Astellas, Gilead, Merck/MSD, and Pfizer. All other authors have no financial conflicts of interest.

c) 2014 Society for Mucosal Immunology

\section{REFERENCES}

1. Bilate, A.M. \& Lafaille, J.J. Induced CD4 + Foxp3 + regulatory T cells in immune tolerance. Annu. Rev. Immunol. 30, 733-758 (2012).

2. Cebula, A., Seweryn, M., Rempala, G.A., Pabla, S.S., Mclndoe, R.A. \& Denning, T.L. et al. Thymus-derived regulatory $T$ cells contribute to tolerance to commensal microbiota. Nature 497, 258-262 (2013).

3. Curotto de Lafaille, M.A., Kutchukhidze, N., Shen, S., Ding, Y., Yee, H. \& Lafaille, J.J. Adaptive Foxp3 + regulatory Tcell-dependent and -independent control of allergic inflammation. Immunity 29, 114-126 (2008).

4. Curotto de Lafaille, M.A. \& Lafaille, J.J. Natural and adaptive foxp3+ regulatory T cells: more of the same or a division of labor?. Immunity 30 626-635 (2009).

5. Josefowicz, S.Z., Niec, R.E., Kim, H.Y., Treuting, P., Chinen, T. \& Zheng, Y. et al. Extrathymically generated regulatory $\mathrm{T}$ cells control mucosal TH2 inflammation. Nature 482, 395-399 (2012).

6. Lathrop, S.K., Bloom, S.M., Rao, S.M., Nutsch, K., Lio, C.W. \& Santacruz, N. et al. Peripheral education of the immune system by colonic commensal microbiota. Nature 478, 250-254 (2011).

7. Bacher, P. \& Scheffold, A. Flow-cytometric analysis of rare antigen-specific T cells. Cytometry A 83, 692-701 (2013).

8. Brown, G.D., Denning, D.W., Gow, N.A., Levitz, S.M., Netea, M.G. \& White, T.C. Hidden killers: human fungal infections. Sci. Transl. Med. 4, 165rv113 (2012).

9. Brakhage, A.A. Systemic fungal infections caused by Aspergillus species: epidemiology, infection process and virulence determinants. Curr. Drug Targets 6, 875-886 (2005).

10. Fröhlich-Nowoisky, J., Pickersgill, D.A., Despres, V.R. \& Poschl, U. High diversity of fungi in air particulate matter. Proc. Natl. Acad. Sci. USA 106, 12814-12819 (2009).

11. Iliev, I.D., Funari, V.A., Taylor, K.D., Nguyen, Q., Reyes, C.N. \& Strom, S.P. et al. Interactions between commensal fungi and the C-type lectin receptor Dectin-1 influence colitis. Science 336, 1314-1317 (2012).

12. Kontoyiannis, D.P. Invasive mycoses: strategies for effective management. Am. J. Med. 125 (1 Suppl), S25-S38 (2012).

13. Knutsen, A.P. \& Slavin, R.G. Allergic bronchopulmonary aspergillosis in asthma and cystic fibrosis. Clin. Dev. Immunol. 2011 843763 (2011).

14. Hohl, T.M., Rivera, A. \& Pamer, E.G. Immunity to fungi. Curr. Opin. Immunol. 18, 465-472 (2006).

15. Brakhage, A.A., Bruns, S., Thywissen, A., Zipfel, P.F. \& Behnsen, J. Interaction of phagocytes with filamentous fungi. Curr. Opin. Microbiol. 13, 409-415 (2010).

16. Romani, L. Immunity to fungal infections. Nat. Rev. Immunol. 11, 275-288 (2011).

17. Wüthrich, M., Deepe, G.S. Jr. \& Klein, B. Adaptive immunity to fungi. Annu. Rev. Immunol. 30, 115-148 (2012).

18. Bacher, P., Schink, C., Teutschbein, J., Kniemeyer, O., Assenmacher, M. \& Brakhage, A.A. et al. Antigen-reactive T cell enrichment for direct, highresolution analysis of the human naive and memory Th cell repertoire. J. Immunol. 190, 3967-3976 (2013).

19. Schoenbrunn, A., Frentsch, M., Kohler, S., Keye, J., Dooms, H. \& Moewes, B. et al. A converse 4-1BB and CD40 ligand expression pattern delineates activated regulatory T cells (Treg) and conventional T cells enabling direct isolation of alloantigen-reactive natural Foxp3 + Treg. J. Immunol. 189, 5985-5994 (2012).

20. de la Rosa, M., Rutz, S., Dorninger, H. \& Scheffold, A. Interleukin-2 is essential for CD4 + CD25 + regulatory Tcell function. Eur. J. Immunol. 34 2480-2488 (2004)

21. Kimmig, S., Przybylski, G.K., Schmidt, C.A., Laurisch, K., Mowes, B. \& Radbruch, A. et al. Two subsets of naive T helper cells with distinct T cell receptor excision circle content in human adult peripheral blood. J. Exp. Med. 195, 789-794 (2002).
22. Geiger, R., Duhen, T., Lanzavecchia, A. \& Sallusto, F. Human naive and memory CD4 + T cell repertoires specific for naturally processed antigens analyzed using libraries of amplified T cells. J. Exp. Med. 206, 1525-1534 (2009).

23. Belkaid, Y., Piccirillo, C.A., Mendez, S., Shevach, E.M. \& Sacks, D.L. CD4 + CD25 + regulatory T cells control Leishmania major persistence and immunity. Nature 420, 502-507 (2002).

24. Belkaid, Y. \& Tarbell, K. Regulatory T cells in the control of hostmicroorganism interactions ( ${ }^{*}$ ). Annu. Rev. Immunol. 27, 551-589 (2009).

25. Shafiani, S., Dinh, C., Ertelt, J.M., Moguche, A.O., Siddiqui, I. \& Smigiel, K.S. et al. Pathogen-specific Treg cells expand early during Mycobacterium tuberculosis infection but are later eliminated in response to Interleukin-12. Immunity 38, 1261-1270 (2013).

26. Acosta-Rodriguez, E.V., Rivino, L., Geginat, J., Jarrossay, D., Gattorno, M. \& Lanzavecchia, A. et al. Surface phenotype and antigenic specificity of human interleukin 17-producing T helper memory cells. Nat. Immunol. 8 , 639-646 (2007).

27. Chai, L.Y., van de Veerdonk, F., Marijnissen, R.J., Cheng, S.C., Khoo, A.L. \& Hectors, M. et al. Anti-Aspergillus human host defence relies on type $1 \mathrm{~T}$ helper (Th1), rather than type 17T helper (Th17), cellular immunity. Immunology 130, 46-54 (2010).

28. Chaudhary, N., Staab, J.F. \& Marr, K.A. Healthy human T-Cell Responses to Aspergillus fumigatus antigens. PLoS ONE 5, e9036 (2010).

29. Zielinski, C.E., Mele, F., Aschenbrenner, D., Jarrossay, D., Ronchi, F. \& Gattorno, M. etal. Pathogen-induced human TH17 cells produce IFN-gamma or IL-10 and are regulated by IL-1beta. Nature 484, 514-518 (2012).

30. Atarashi, K., Tanoue, T., Shima, T., Imaoka, A., Kuwahara, T. \& Momose, Y. et al. Induction of colonic regulatory $T$ cells by indigenous Clostridium species. Science 331, 337-341 (2011).

31. Holt, P.G., Strickland, D.H., Wikstrom, M.E. \& Jahnsen, F.L. Regulation of immunological homeostasis in the respiratory tract. Nat. Rev. Immunol. 8, 142-152 (2008).

32. Maloy, K.J. \& Powrie, F. Intestinal homeostasis and its breakdown in inflammatory bowel disease. Nature 474, 298-306 (2011).

33. Round, J.L. \& Mazmanian, S.K. Inducible Foxp3 + regulatory T-cell development by a commensal bacterium of the intestinal microbiota. Proc. Natl. Acad. Sci. USA 107, 12204-12209 (2010).

34. Murdock, B.J., Shreiner, A.B., McDonald, R.A., Osterholzer, J.J., White, E.S. \& Toews, G.B. et al. Coevolution of TH1, TH2, and TH17 responses during repeated pulmonary exposure to Aspergillus fumigatus conidia. Infect. Immun. 79, 125-135 (2011).

35. Cheng, S.C., Joosten, L.A., Kullberg, B.J. \& Netea, M.G. Interplay between Candida albicans and the mammalian innate host defense. Infect. Immun. 80, 1304-1313 (2012).

36. Aimanianda, V., Bayry, J., Bozza, S., Kniemeyer, O., Perruccio, K. \& Elluru, S.R. et al. Surface hydrophobin prevents immune recognition of airborne fungal spores. Nature 460, 1117-1121 (2009).

37. Bruns, S., Kniemeyer, O., Hasenberg, M., Aimanianda, V., Nietzsche, S. \& Thywissen, A. et al. Production of extracellular traps against Aspergillus fumigatus in vitro and in infected lung tissue is dependent on invading neutrophils and influenced by hydrophobin RodA. PLoS Pathog. 6, e1000873 (2010).

38. Gottschalk, R.A., Corse, E. \& Allison, J.P. TCR ligand density and affinity determine peripheral induction of Foxp3 in vivo. J. Exp. Med. 207, 1701-1711 (2010).

39. Graca, L., Chen, T.C., Le Moine, A., Cobbold, S.P., Howie, D. \& Waldmann, H. Dominant tolerance: activation thresholds for peripheral generation of regulatory T cells. Trends Immunol. 26, 130-135 (2005).

40. Kretschmer, K., Apostolou, I., Hawiger, D., Khazaie, K., Nussenzweig, M.C. \& von Boehmer, H. Inducing and expanding regulatory Tcell populations by foreign antigen. Nat. Immunol. 6, 1219-1227 (2005).

41. Soroosh, P., Doherty, T.A., Duan, W., Mehta, A.K., Choi, H. \& Adams, Y.F. et al. Lung-resident tissue macrophages generate Foxp3 + regulatory $\mathrm{T}$ cells and promote airway tolerance. J. Exp. Med. 210, 775-788 (2013).

42. Floess, S., Freyer, J., Siewert, C., Baron, U., Olek, S. \& Polansky, J. et al. Epigenetic control of the foxp3 locus in regulatory Tcells. PLoS Biol. 5, e38 (2007).

43. Hoffmann, P., Boeld, T.J., Eder, R., Huehn, J., Floess, S. \& Wieczorek, G. et al. Loss of FOXP3 expression in natural human CD4 + CD25+ regulatory $\mathrm{T}$ cells upon repetitive in vitro stimulation. Eur. J. Immunol. 39, 1088-1097 (2009). 


\section{ARTICLES}

44. Chaudhary, N. \& Marr, K.A. Impact of Aspergillus fumigatus in allergic airway diseases. Clin. Transl. Allergy 1, 4 (2011).

45. Donnelly, L.E. \& Barnes, P.J. Defective phagocytosis in airways disease. Chest 141, 1055-1062 (2012).

46. Duan, W., So, T., Mehta, A.K., Choi, H. \& Croft, M. Inducible CD4+ LAP + Foxp3- regulatory $T$ cells suppress allergic inflammation. J. Immunol. 187, 6499-6507 (2011).

47. Lloyd, C.M. \& Hawrylowicz, C.M. Regulatory T cells in asthma. Immunity 31, 438-449 (2009).

48. Ostroukhova, M., Seguin-Devaux, C., Oriss, T.B., Dixon-McCarthy, B., Yang, L. \& Ameredes, B.T. et al. Tolerance induced by inhaled antigen involves CD4(+) T cells expressing membrane-bound TGF-beta and FOXP3. J. Clin. Invest. 114, 28-38 (2004).
49. Ohshima, M., Futamura, M., Kamachi, Y., Ito, K. \& Sakamoto, T. Allergic bronchopulmonary aspergillosis in a 2-year-old asthmatic boy with immune dysregulation, polyendocrinopathy, enteropathy, X-linked. Pediatr. Pulmonol. 44, 297-299 (2009).

50. Hebart, H., Bollinger, C., Fisch, P., Sarfati, J., Meisner, C. \& Baur, M. et al. Analysis of T-cell responses to Aspergillus fumigatus antigens in healthy individuals and patients with hematologic malignancies. Blood 100, 4521-4528 (2002).

51. Wieczorek, G., Asemissen, A., Model, F., Turbachova, I., Floess, S. \& Liebenberg, V. et al. Quantitative DNA methylation analysis of FOXP3 as a new method for counting regulatory T cells in peripheral blood and solid tissue. Cancer Res 69, 599-608 (2009). 